\title{
Analysis of Hardware Architecture Markovian Chain Queuing Model for Wireless Sensor Networks
}

\author{
Mabruka Mohamed Agel*, Mohamed Hadi Habaebi and B. A. Hamida \\ Electrical and Computer Engineering Department, Faculty of Engineering, \\ International Islamic University Malaysia (IIUM) \\ mabrouka9999@gmail.com,habaebi@iium.edu.my, belal@iium.edu.my
}

\begin{abstract}
A wireless Sensor Network (WSN) commonly consumes fairly less energy whereas light and multiple-distributed sensors along with an appropriate wireless network usually come with a lower data rate. An increased system performance along with relatively a higher stability can be achieved by means of applying suitable routing protocol and QoS. QoS represents the effectiveness and robustness of any system concerned. The expansion in relation to productivity of a system may depend on keeping the energy consumption at a controlled level. This paper reports on a study in which quality measurement service was provided by means of analyzing packets in the queuing systems by employing queuing theory. The proposed model is two M/M/1/N queues in tandem with $N$ buffer capacity. The simulation which was performed by MATLAB software indicated that an increase in the service rate of one of the servers while fixing the service rate of another server may result in a waste of hardware resources. Also, the blocking probability for same buffer's size and data arrival rate will not reach less than specific values.
\end{abstract}

Keywords: Blocking probability, Quality of service, Queuing theory, Wireless sensor ${ }^{1}$ networks.

\section{Introduction}

A Wireless Sensor Network (WSN) is a system device which is embedded and selfconfigured to serve the purpose of monitoring physical or environmental conditions such as temperature, sound, motion or pollutants. In addition, it also cooperatively passes its data by means of the network to a main location or sink in which the data are usually observed and analyzed. [1]

Considering the fact that wireless sensor networks are scalable, therefore they require very little energy to consume. Notably, they are also considered efficient, programmable and cost relatively less to purchase and install. More importantly, they require almost zero maintenance. It is noteworthy that in traditional sensor networks, the packet size is considerably smaller which therefore makes it easier to deliver from the source node to the destination node. In the case of multimedia data for which its size is measured in megabytes, it is therefore quite a challenge for data transfer. Consumption of energy in wireless sensor network remains a major problem to date. It is worth noting that an increase in hardware specifications may result in energy overconsumption. [2]One of the possible ways of extending the life of the network is adding energy supplies such as solar cells. Notwithstanding, such additions may have implications on the cost of sensor nodes, and thus may result in pressure on application areas.

Despite the challenges which have been discussed in the foregoing, there must be a trade-off between energy consumption and the use of resources. In the event of the data

* Corresponding Author: Mohamed Hadi Habaebi ECE Dept. Faculty of Engineering, IIUM 
which need to be processed exceeding the capacity of the system, data loss and dropping may have to be experienced. Although the loss of audio and video packets may not seriously affect the usability of the data owing partly to the packets still remaining recognizable to convey the message to the listeners, there are however some limits in relation to the data loss which when surpassed, the delivered messages can be corrupted and therefore beyond recognition. [3] Thus, an efficient utilization of the available resources to sensor nodes (e.g. energy supply and bandwidth limitation) is no longer considered an extravagance but a necessity. The energy consumption involved in transmitting higher volume of data is quite significant. As the energy is generated by sensor nodes, it is therefore imperative to address these issues at the routing layer. [2]

Beside energy efficiency, other quality of service (QoS) necessities of throughput, accepted packet delay, packet loss, ought to be considered during the design process of sensor networks. [4-5] The buffer's size of a sensor hub usually is a constraint. Consequently, appropriately picking the size of the buffer is also critical in planning sensor networks [6]. Buffer overloading leads to loss in data which requires retransmission of the same packets many times causing additional energy consumption. Hence, queuing process is connected to energy consumption.

A few queuing systems like production lines which are referred to as classical networks have known distribution probabilities therefore algorithms can be utilized to determine the throughput of the system. Classical systems assume that the capacity of the serving stations is limitless, a supposition that often does not meet the specifications of queuing systems in real life in the particular computer and networking systems. This introduces the concept of queuing systems with blocking. [7]

A fairly well-known design of queuing systems with blocking is that the tandem configuration investigated in this paper. Tandem queuing system is an open queuing system comprising of size limited queues in series. Basically, a tandem queuing framework comprises of a number of servers in series with buffers located between every two successive servers. In spite of the simplicity of the structure of the system, its performance is very complex. Basically, tandem queuing networks with limited buffers are seen as a class of stochastic dynamic systems. The movement of units through one line is at risk of being blocked when the capacity limit of another buffer is reached. [8]

This paper attempts to elaborate the use of queuing theory in managing wireless sensor networks by studying different parameters which are believed to affect the efficiency of these networks. In this regard, a comprehensive review of the previous related works and their salient findings along with the approaches employed are summarized in section (II). Moving on, section (III) delineates the proposed Hardware design of WSN which is considered for analyzing similar systems. Related models which have widely been used in studying WSN queuing systems are presented in section (IV). What follows next is the findings along with the analysis of the system's outcomes are presented in Section (V). Next, some potential conclusions which can be drawn from the discussion of this paper is reported in section (VI). Lastly, section (VII) lists the studies which were referred to in reporting the present study.

\section{Related Works}

The management of queuing systems has widely been studied by many researchers across various contexts. For instance, related constraints encountered in designing wireless sensor networks were investigated in [9]. Listing the cost of production as one of the parameters that should be taken into consideration as a trade-off must be made between both the cost and hardware specifications. Notably, the optimization of hardware specifications may only come with increments in both the cost of production along with more energy consumption. 
In this regard, it is worth noting that Queuing may help provide better services and therefore achieve optimum efficiency in relation to offering services. Presently, technology is capable of providing designed electro-mechanical and computerized systems which may help control and manage queues. [7-8] In relation to the node at which the wireless network is placed, it is relatively harder to modify the hub's sensors accommodating to particular needs. Therefore, related studies have called for a stable large-scale WSN hubs modelling techniques [10]. Upon carefully assessing the distributed hubs, potentially ideal parameters required for the modelling of the hubs' hardware have been identified. The proposed strategy in view of Markovian model in [11-13] can be considered more appropriate for macro scale WSN. However, it was not particularly designed for large-scale wireless sensor networks.

Queuing network can be considered as a compelling and operational method for designing and modelling systems. It is designed as part of the demonstrating, designing and modelling the analysis involved in processing and transmission systems [14-15]. It reportedly offers numerous benefits which may incorporate exceeding theoretical and mathematical aspects in relation to designs and therefore perform the modelling processes. Various communication and transmission protocols are involved for wireless sensor network systems, including the ZigBee. ZigBee is reportedly the main worldwide standard which has widely been referred to for minimal cost, low-data rate, and short medium range remote sensing systems with relatively lower battery consumption. [16] In addition, sophisticated controlling systems are also considered necessary for commercial and mechanical applications. In a wireless sensor system which is intended for industry, office, smart home or other applications, considerably higher number of gadgets are therefore interconnected and managed using wireless sensors [4, 17]. The enormous number of gadgets is placed within the system field for effectively attaining the required data and the phenomenon under investigation was studied.

Various studies to date have already been carried out on the diverse wireless network, outline, topology and modelling for the expansion of remote systems and QoS exploration points of view [18, 19]. For instance, a related study [20] discussed the QoS management for a wireless system in relation to the radio ranges of such networks.

The researchers in $[18,20]$ have proposed wireless sensor node MAC modelling protocols for evaluating the performance and transmission process. Another related study [21] explored un- buffered queues of Multimedia sensor nodes with regards to the quality of service (QoS) and the relationship between data processing rates and blocking rates. Notwithstanding, no mentions were made in relation to the analysis of buffer configuration and their impact on the QoS. It was shown that for a tandem queuing system, in which the optimization is performed on bufferless network, it is of utmost importance to strike a balance between the processing capabilities and cost involved for the system to achieve the best performance.

\section{Hardware Architecture Reference Design}

According to [22], the hardware architecture refers to Sensor nodes in WSNs which are embedded with low-cost sensor boards. Sensors usually remain inactive and they may only act upon the detection of intruders or any unusual environmental phenomenon. When certain actions need to be monitored, it is only then they are activated. However, it is worth noting that the wireless sensor device is considered alert at all times regardless of whether there is an intrusion or not. [23].

In the literature, multiple results on analysing queuing systems of remote sensor networks have been presented. A classic approach (as described in [24, 25]) in WSN's queuing systems is displaying a sensor hub as a finite FIFO queue modelled by Markov's chain. 
In this paper, a modelling of remote sensor systems is presented. Every sensor hub is demonstrated as an $\mathrm{M} / \mathrm{M} / 1 / \mathrm{N}$ queue. A major assumption of this paper is that all data are composed into packets with a variable length with exponential distribution. Data collecting process at every sensor hub follows a Poisson distribution assuming data collecting events are independent from each other. Every end node transmits its collected data to the main sink. [26]

The model proposed here is intended to reproduce the processing and transmitting stages of collected data at the end nodes of remote sensor systems. In [27] an IEEE 1451.5 data colleting system was introduced, where data was gathered utilizing Wireless Transmission Sensor Interface Module (WTIM), which then sent for processing via the network capable application processor (NCAP). The primary buffer was situated within the processing unit for collected data and also the second buffer was located in the transmission unit to store the data to be transmitted.

The model proposed by this paper will outline parameters like the average length of a queue and sojourn times at server. Since the system is in steady state if and only if the number of units in the primary queue remains limited, so the maximum throughput can be easily calculated. Finally, the scope of this paper will be the realtime service blocking queuing systems such as data sensing networks.

The design shown in the form of a diagram in Figure 1 is based on WSN data acquisition system. As can be seen in the diagram, the data were collected from the sensor. These data were then passed to the processing unit at which the packets were kept in buffer pending their turns. The processed data were sent next to the transmission unit at which the data were put into packets and transferred wirelessly. [27]

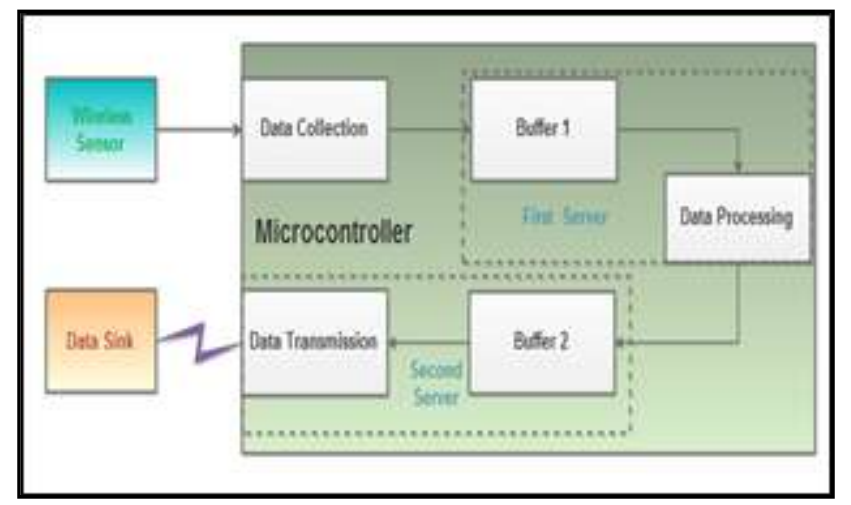

\section{Figure 1. Schematic Diagram of a Queuing System of Wireless Sensor Network}

\section{Markovian Chain Queuing Model}

In this paper, the management of a system consisting of two servers was studied by two models of which, the first model was with buffer-less servers and the second model was with a more generalized system which consisted of two buffered servers.

\subsection{Performance Analysis of Buffer-Less Servers Queuing Model}

A comprehensive review of literature indicated that a buffer-less model for studying queuing systems in wireless sensor network was proposed in [22]. The system is made up of two servers i.e., Data processor as a server, data transmitter as the second server. It should be noted that an arriving packet may first go to server 1 . Upon being processed, it may either be passed to server 2 if that server is unoccupied or else, it has to wait for server 1 which is being occupied until it is available. Notably, packets usually enter server1 as long as it is readily available. 
In relation to this model, potential packets arrive in accordance with the Poisson rate $\lambda$ rate and exponential service rates of $\mu 1$ and $\mu 2$. Five states of this system that reflect the number of packets at each server; these states are $(0,0),(1,0),(0,1),(1,1),(b, 1)$ as has been shown in the transition diagram shown in figure 2. [1]

State $(b, 1)$ represents the case in which there is a packet in server 1 which has already been processed but wait in server1 due to the fact that there is presently a packet being processed in server 2, i.e., a packet blocking the other packets from entering the whole system. This leads to the balance equations of the system illustrated in table 1 .

When the queue in equilibrium the summation of states equals 1 :

$$
\pi_{00}+\pi_{10}+\pi_{11}+\pi_{b 1}+\pi_{01}=1
$$

$$
\pi_{00}=\frac{1}{1+\frac{\lambda}{\mu_{1}}\left(1+\frac{\lambda}{\left(\mu_{1}+\mu_{2}\right)}\right)+\frac{\lambda^{2}}{\mu_{2}\left(\mu_{1}+\mu_{2}\right)}+\frac{\mu_{1} \lambda^{2}}{\mu_{2}^{2}\left(\mu_{1}+\mu_{2}\right)}+\frac{\lambda}{\mu_{2}}}
$$

Table 1. Summary of States in the Buffer-Less Model Proposed By [22]

\begin{tabular}{|c|c|}
\hline State & Balance Equation \\
\hline$(0,0)$ & Rate at which the process leaves $=$ rate at which it enters \\
\hline$(1,0)$ & $\lambda \pi_{00}=\mu_{2} \pi_{01}$ \\
\hline$(0,1)$ & $\mu_{1} \pi_{10}=\lambda \pi_{00}+\mu_{2} \pi_{11}$ \\
\hline$(1,1)$ & $\left(\lambda+\mu_{2}\right) \pi_{01}=\mu_{1} \pi_{10}+\mu_{2} \pi_{b 1}$ \\
\hline$(b, 1)$ & $\left(\mu_{1}+\mu_{2}\right) \pi_{11}=\lambda \pi_{01}$ \\
\hline$\mu_{2} \pi_{b 1}=\mu_{1} \pi_{11}$ \\
\hline
\end{tabular}

Blocking probability is the sum of the all the probabilities of the states in which no packet can enter the system are: $\pi_{10}, \quad \pi_{11}$ and $\pi_{\text {b1 }}$.

$$
\mathrm{P}_{\mathrm{b}}=\left[\frac{\lambda}{\mu_{1}}\left(1+\frac{\lambda}{\left(\mu_{1}+\mu_{2}\right)}\right)+\frac{\lambda^{2}}{\mu_{2}\left(\mu_{1}+\mu_{2}\right)}+\frac{\mu_{1} \lambda^{2}}{\mu_{2}^{2}\left(\mu_{1}+\mu_{2}\right)}\right]
$$

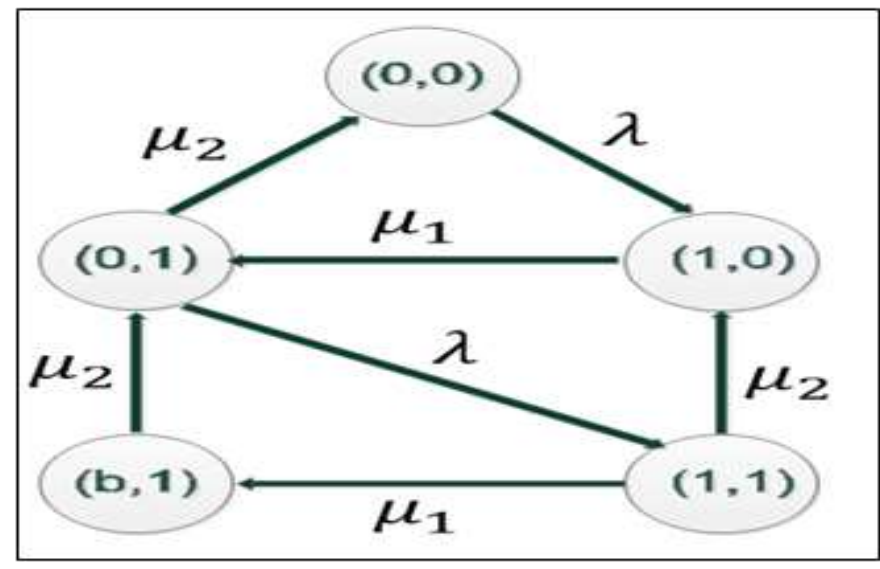

Figure 2. Transition Diagram for the Buffer-Less Model Proposed by [22]

It is notable that determining the average number of packets within the system is a relatively easier task considering that a maximum of two packets can be in the system for the states $(1,1)$ or $(b, 1)$, and only one packet for the states $(01)$ or $(1,0)$. Therefore, the average number of packets in the system is:

$\mathrm{L}=\pi_{01}+\pi_{10}+2\left(\pi_{11}+\pi_{\mathrm{b} 1}\right)$ 
Another metric which can be used for evaluating the system is the average time of a packet spent in the system which can be discovered by means of little's theorem. In this model, packets may enter the system only by means of the states $(0,0)$ or $(0,1)$. Thus, the average amount of time that an entering packet may possibly spend in the system is explained by:

$W=\frac{\pi_{01}+\pi_{10}+2\left(\pi_{11}+\pi_{b 1}\right)}{\lambda\left(\pi_{00}+\pi_{01}\right)}$

\subsection{Performance Analysis of Buffered Servers Queuing Model}

The network consists of two single server queues. The service time at each queue may follow an exponential distribution with rate $\mu \mathrm{i}$ and the external arrivals to queue 1 are independent of the Poisson processes with rate $\lambda$. In this regard, the packets may follow the FIFO queuing method. In other words, any packet which arrives when the queues are full is denied service. The probability of a packet being processed is $\left(1-\mathrm{P}_{\mathrm{N}}\right)$.In analyzing this model in the present study, the following assumptions were made:

- $\quad$ Packets arrive to queue 1 with Poisson distribution with effective rate $\lambda_{1}{ }_{1}$.

- $\quad$ Upon completion of service at server 1, the packet may move on to server 2 as long as the first queue is not blocked by the second one. When queue 1 is blocked by queue 2 , it is assumed that there is no arrival from queue 1 to queue 2 at all.

- $\quad$ The service time is exponentially distributed.

- $\quad$ A packet at server 1, upon completion the service, will deal with the destination queue as it is in a steady state.

- $\quad$ The moment a packet arrives at the tandem system, the second server will become an $\mathrm{M} / \mathrm{M} / 1 / \mathrm{N}$ finite waiting capacity queue with an overall arrival rate which equals the service rate of the first server (the single-node approximation).

- $\quad$ The arrival rate at the second server is Poisson for $\mu_{1}>\lambda$, and the arrival rate at the second server is exponentially distributed $\mu_{1}$ for $\mu_{1}<\lambda$, the model still Markovian and $\mathrm{M} / \mathrm{M} / 1 / \mathrm{N}$ formula applies. The states diagram is shown in figure (3).

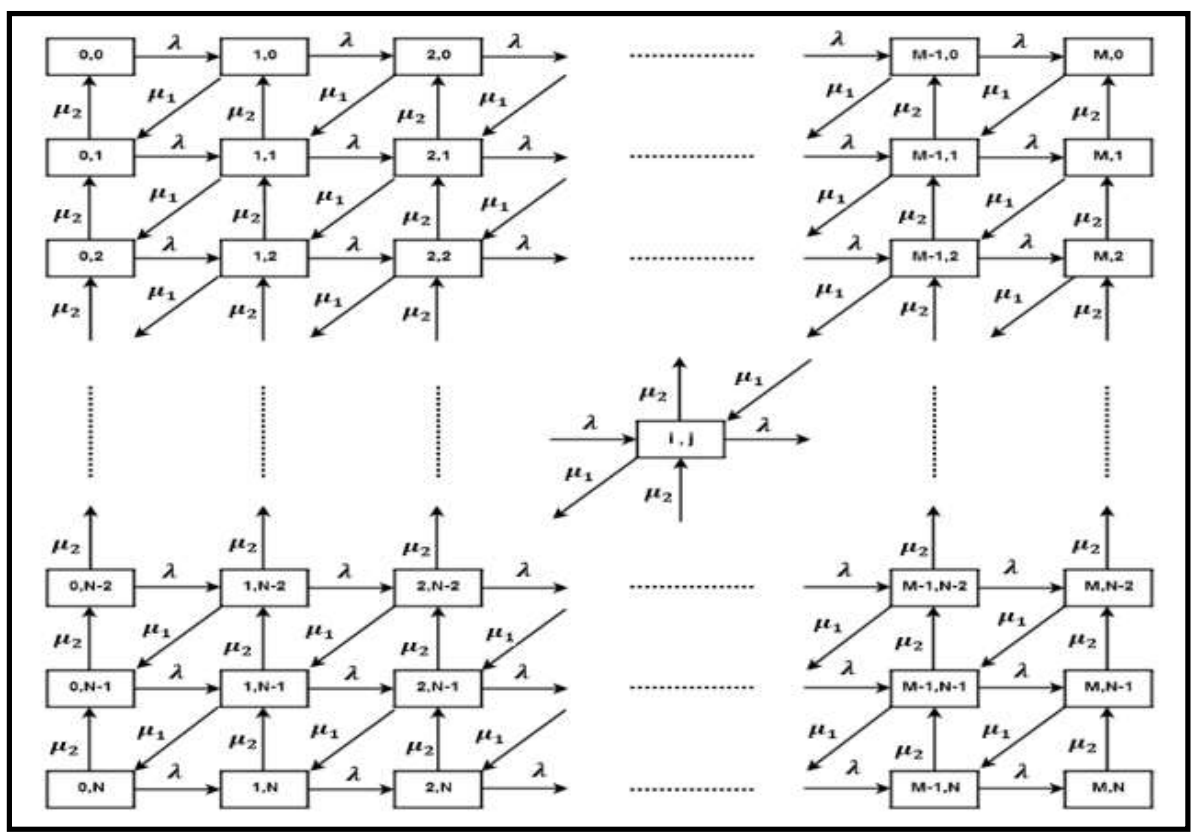

Figure 3. Two-Dimensional Markov Chain State Diagram of Two Tandem Servers Network 
The balance equations associated with the steady-state probabilities of the system states are as follows:

$$
\begin{aligned}
& \lambda \mathrm{p}_{(0,0)=} \mu_{2} \mathrm{p}_{(0,1)} \\
& \left(\lambda+\mu_{1}\right) \mathrm{p}_{(\mathrm{i}, 0)=\mu_{2}} \mathrm{p}_{(1, j)}+\lambda \mathrm{p}_{(\mathrm{i}-1,0)} \text {; for } 1 \leq \mathrm{i}<\mathrm{M} \\
& \left(\lambda+\mu_{2}\right) \mathrm{p}_{(0, \mathrm{j})=} \mu_{1} \mathrm{p}_{(1, \mathrm{j}-1)}+\mu_{2} \mathrm{p}_{(0, \mathrm{j}+1)} ; \text { for } 1 \leq \mathrm{j}<\mathrm{N} \\
& \left(\lambda+\mu_{2}\right) \mathrm{p}_{(\mathrm{i}, \mathrm{N})}=\mu_{1} \mathrm{p}_{(\mathrm{i}+1, \mathrm{~N}-1)}+\lambda \mathrm{p}_{(\mathrm{i}-1, \mathrm{~N})} \text {; for } 0 \leq \mathrm{I} \\
& \left(\mu_{1}+\mu_{2}\right) \mathrm{p}_{(\mathrm{M}, \mathrm{j})}=\lambda \mathrm{p}_{(\mathrm{M}-1, \mathrm{j})}+\mu_{2} \mathrm{p}_{(\mathrm{M}, \mathrm{j}+1)} ; \text { for } 1 \leq \mathrm{j} \\
& \left(\lambda+\mu_{1}+\mu_{2}\right) \mathrm{P}_{(\mathrm{i} i \mathrm{j})=}=\lambda \mathrm{P}_{(\mathrm{i}-1, \mathrm{j} j \mathrm{j}}+\mu_{1} \mathrm{P}_{(\mathrm{i}+1, \mathrm{j}-1)}+\mu_{2} \mathrm{P}_{(\mathrm{i} j \mathrm{j}+1)} \text {; for } 1 \leq \mathrm{i}<\mathrm{M} \text { and } 1 \leq \mathrm{j}<\mathrm{N}
\end{aligned}
$$

The capacity of each server within the system is considered limited to only $\mathrm{N}$ packets. When there is a number of jobs which equal that capacity, the entering rate to the server can therefore be zero and no packets can be allowed to enter the server and be processed till the previous packet fully accomplished its job and exits the server eventually. [28]

The blocking probability of such a server with $\mathrm{N}$ packets equals;

$$
\mathrm{p}_{\mathrm{N}}=\rho^{\mathrm{N}} \frac{1-\rho}{1-\rho^{\mathrm{N}+1}}
$$

In this regard, the effective arrival rate (Packets which enter the system are served) is as follows:

$$
\lambda_{\text {eff }}=\lambda\left(1-\mathrm{p}_{\mathrm{N}}\right)
$$

Thus, the average number of packets in the server is shown in the following equation:

$$
L_{a}=\rho\left(\frac{1-(\mathrm{N}+1) \rho^{\mathrm{N}}+\mathrm{N} \rho^{\mathrm{N}+1}}{(1-\rho)\left(1-\rho^{\mathrm{N}+1)}\right.}\right) ; \rho \neq 1
$$

Consequently the average sojourn time of a packet in the server, Ws, is:

$$
W_{\mathrm{s}}=\frac{\mathrm{L}_{\mathrm{g}}}{\lambda_{\mathrm{eff}}}=\frac{\mathrm{L}_{\mathrm{g}}}{\lambda\left(1-\mathrm{pN}_{\mathrm{N}}\right)}
$$

The above-mentioned formulas can be used to evaluate $2 \mathrm{M} / \mathrm{M} / 1 / \mathrm{N}$ queues in series as is the case in the present model for the second queue.

When: $\lambda_{\text {eff1 }} \geq \mu_{1}, \lambda_{2}=\mu_{1}$ and if $\lambda_{\text {eff1 }} \leq \mu_{1}, \lambda_{2}=\lambda_{\text {eff } 1}$ and $\lambda_{\text {eff2 }}=\lambda_{2}\left(1-p_{\mathrm{N}}\right)$

Combining the two servers as one tandem queue will produce the model under the study. Thus, the total blocking probability is as shown in the following:

$$
\mathrm{P}_{\mathrm{b}}=\mathrm{p}_{\mathrm{M}} \cup \mathrm{p}_{\mathrm{N}}=\mathrm{p}_{\mathrm{M}}+\mathrm{p}_{\mathrm{N}}-\mathrm{p}_{\mathrm{M}} \times \mathrm{p}_{\mathrm{N}}
$$

Where $\mathrm{P}_{\mathrm{M}}$ and $\mathrm{P}_{\mathrm{N}}$ are independent events

$$
P_{b}=\rho_{1}{ }^{\mathrm{M}} \rho_{2}{ }^{\mathrm{N}} \frac{\left(1-\rho_{1}\right)\left(1-\rho_{2}\right)}{\left(1-\rho_{1} \mathrm{M}+1\right)\left(1-\rho_{2} \mathrm{~N}+1\right)}
$$

In case the buffer's size of server 1 equals buffer's size of server 2; $\mathrm{M}=\mathrm{N}$ then:

$$
P_{b}=\left(\rho_{1} \rho_{2}\right)^{\mathrm{N}} \frac{\left(1-\rho_{1}\right)\left(1-\rho_{2}\right)}{\left(1-\rho_{1}{ }^{N+1}\right)\left(1-\rho_{2}{ }^{N+1}\right)}
$$

When the first buffer is full then packets will be dropped and blocked from entering the system and that does not depend on the event if buffer 2 is full or not as packets will not enter the system at all. When packets exit from server 1 it will arrive at server 2 , if the 
server is blocked then it will be blocked also and not served at server 2 regardless if server 1 is blocked or not.

$$
\begin{aligned}
& \mathrm{L}_{\mathrm{g}}=\mathrm{L}_{\mathrm{g} 1}+\mathrm{L}_{\mathrm{g} 2} \\
& \mathrm{~W}_{\mathrm{g}}=\mathrm{W}_{\mathrm{g} 1}+\mathrm{W}_{\mathrm{g} 2}
\end{aligned}
$$

\section{Results and Findings}

The models discussed in this study were executed and simulated by MATLAB software. By means of looking into each of the performance characteristics of the blocking probability, the number of packets in the system and the average sojourn time taken for each packet in the system, the results of the study were obtained.

\subsection{System's Evaluation under Buffer-less Servers' Model}

The impact resulting from an increase in the service rate of the first server whereas the service rate of the second server's service rate remaining constant can be seen in figure 4 . The blocking probability is significantly dropping when an increase is observed in the rate $\mu_{1} / \mu_{2}$, till it becomes almost constant after $\mu_{1}=5 \mu_{2}$. The effect of increasing the arrival rate is also shown in the graph, which indicates that a higher arrival rate may results in a higher blocking probability.

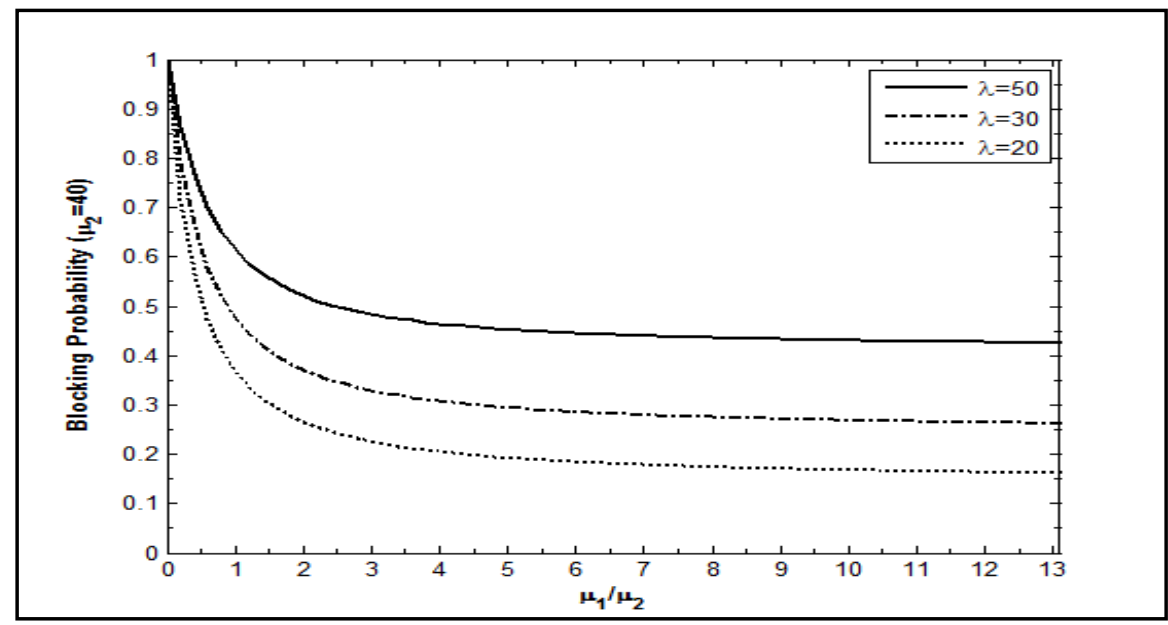

\section{Figure 4. Blocking Probability as a Function of $\mu_{1} / \mu_{2}$ for the Buffer-Less Model}

Similarly, any increase in the service rate $\mu_{2}$ for constant value of $\mu_{1}$ may therefore result in a reduction of the blocking probability. However, such a pattern is only observed till the point of $\mu_{1}=\mu_{2}$, after which, the blocking probability may turn into a constant value as illustrated in figure 5 . 


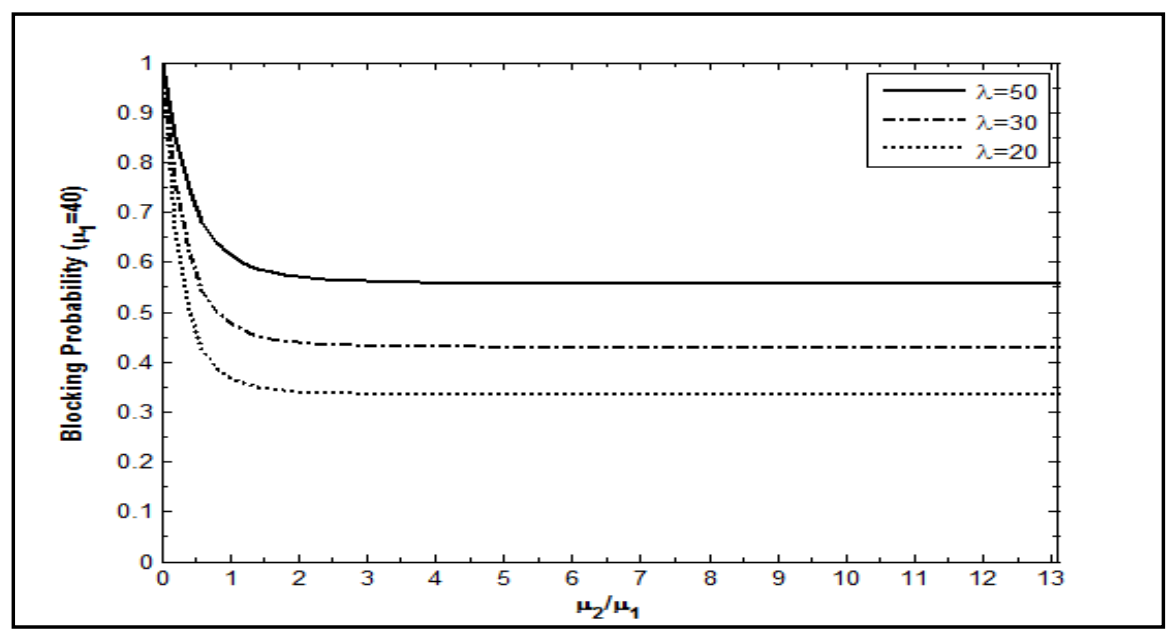

Figure 5. Blocking Probability vs. $\mu_{2} / \mu_{1}$ for the Buffer-Less Model

Thus, in general, any increase observed in the service rate for either of the services results in less blocking being observed in the system. However, if a trade-off must be made on in relation to deciding which should be increased while making the other constant, preferably, the choice should point to increase $\mu_{1}$. Considering the finding which is shown by the graph, for instance, it can be argued that for the same value of arrival rate of $\mu_{1}=2 \mu_{2}$, the blocking probability equals 0.2 . On the other hand, for the case in which $\mu_{2}=2 \mu_{1}$, the blocking probability is 0.34 . These findings are notably in conflict with those of [22], in which it was reported that an increase in the value of $\mu_{1}$ for constant $\mu_{2}$ resulted in higher blocking probability even though similar equations and models were employed.

In attempting to study the effect of $\mu_{1} / \mu_{2}$ on the average number of packets in the system, figure 6 was produced. When $\lambda>\mu_{2}$, the average number of packets will increase up to the value of $\mu_{1}=\mu_{2}$, after which, an increase in the service rate at server 1 and a decrease in blocking probability can be observed respectively. Such a development causes the number of packets to decrease accordingly. In the case of $\lambda<\mu_{2}$, as $\mu_{1}$ increases, the average number of packets will in turn significantly decrease. In all cases, the average number of packets will reach a constant value after $\mu_{1}=\mu_{2}$.

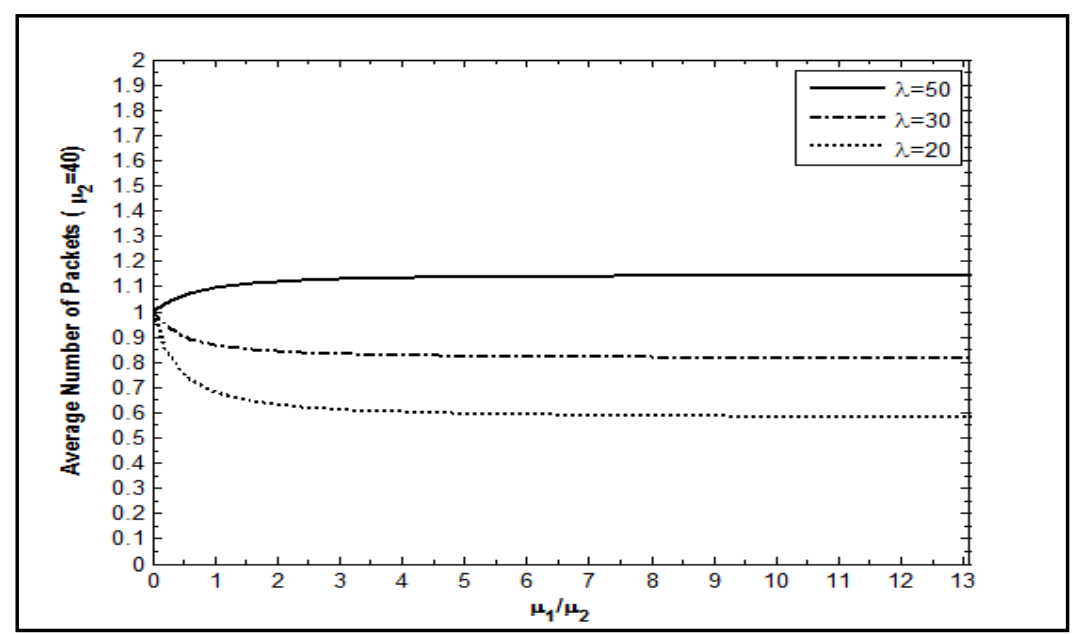

Figure 6. Average Number of Packets Vs. $\mu_{1} / \mu_{2}$ for the Buffer-Less Model

This is also the case where $\mu_{2}$ changes over $\mu_{1}$ (Figure 7), the number of packets keep decreasing up to the value of $\mu_{2}=\mu_{1}$, at which, it may become a constant value. 


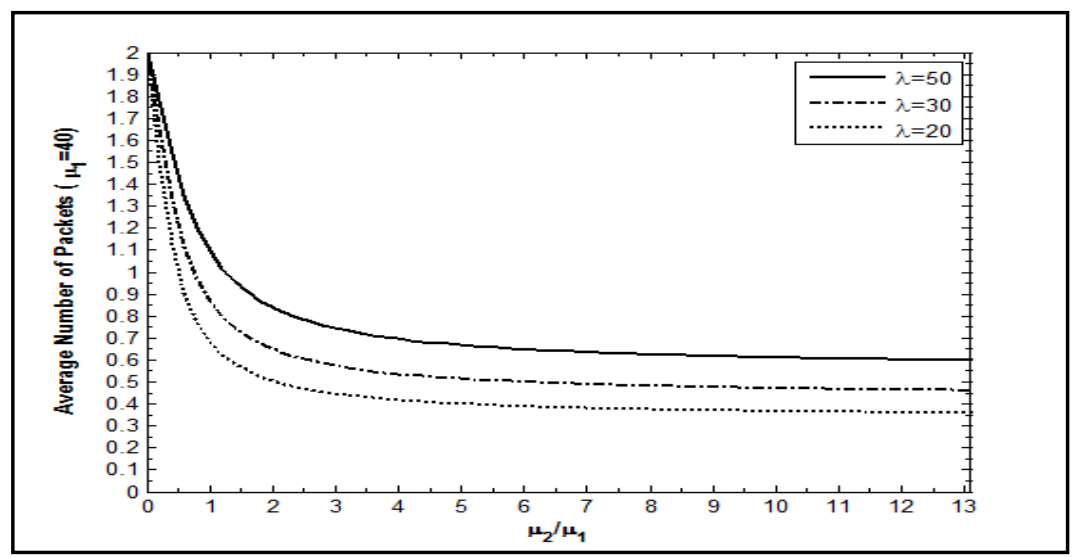

Figure 7. Average Number of Packets as a Function of $\mu 2 / \mu 1$ for the Bufferless Model

\subsection{System's Performance under Buffered Servers' Model}

The motive behind this model is to survey the performance of tandem queuing networks by examining the effect of arrival rates into the input queue on the whole system. Consequently, determining the point where the system is better utilized and finding the balance between the blocking rates and system's cost. This resembles finding the highest achievable throughput of the system. In order to reliably be able to evaluate the proposed model, the impact of different parameters was thus investigated. Three characteristics were investigated under the present study: the blocking probability, average sojourn time and average number of packets in the system. These performance metrics were evaluated against the arrival rate within the system, the service rate observed for both servers and the size of the buffer of the two servers.

5.2.1. The Effect of Buffer's Size: The variation observed in buffer's size and its impact on the blocking probability of the system is shown in figure (8). The change of blocking probability was analysed for different values of $\lambda, \mu_{1}$ and $\mu_{2}$.In general, it can be hypothesized that any increase in the buffer's size may produce less blocking in the system. For a similar value of arrival rate, the change of values of both $\mu_{1}$ and $\mu_{2}$ may not indicate big differences with regards to the trend of the graph although the blocking probability for $\mu_{1}>\mu_{2}$ can be higher than the case in which $\mu_{1}<\mu_{2}$. Such variation can be expected as for the second server $\mu_{1}$ have performed as the arrival rate. 


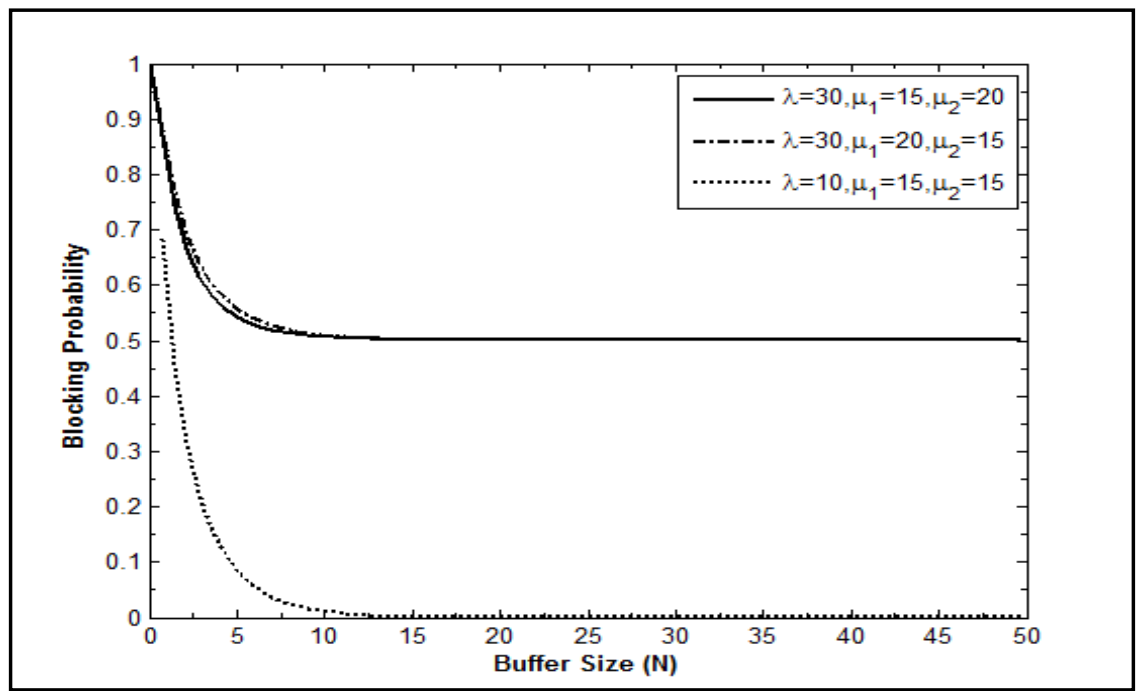

Figure 8. Blocking Probability against Buffer's Size (N)

The lowest blocking probability can be achieved for values where $\lambda<\mu_{1}$ and $\mu_{2}$, and as the size of the buffer increases the system's approaches, the performance of M/M/1 system at a steady state. Similar to the effect of buffer's size on the blocking probability of the system, any increase in buffer's size will increase the average number of packets in the system as can be seen in figure (9).

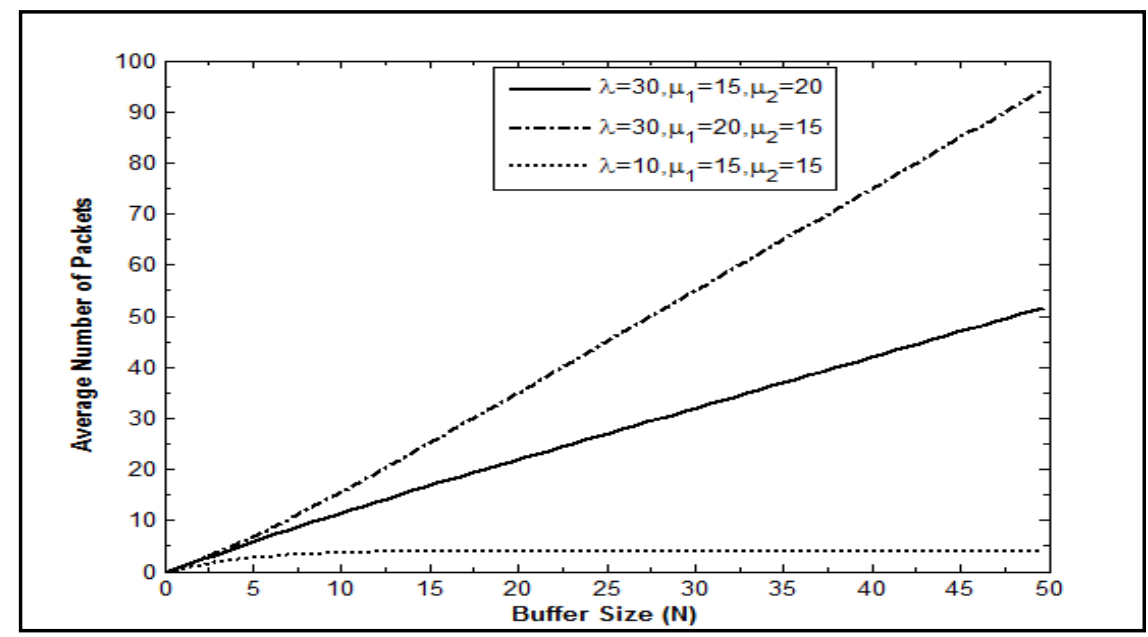

Figure 9. Average Number of Packets in the System vs. Buffer's size

When the arrival rate is observed to be higher than the service rate, the number of packets in the system will continue to increase till the buffers are full. However, in the case of the arrival rate being less than the service rate, the average number of customers can be considered mostly being constant at a very low level. It is noteworthy that a higher service rate makes the packets exiting the system in a very short span of time to ensure that the buffer will never be full.

Due to the fact that the number of packets in the system may affect the average total time taken for a packet in the system, figure 10 shows that increasing the buffer's size may increase the average time spent by a packet in the system. In a similar manner, for the average number of packets in the system, the average sojourn time taken is considered almost constant as the arrival rate being less than the service rate. 


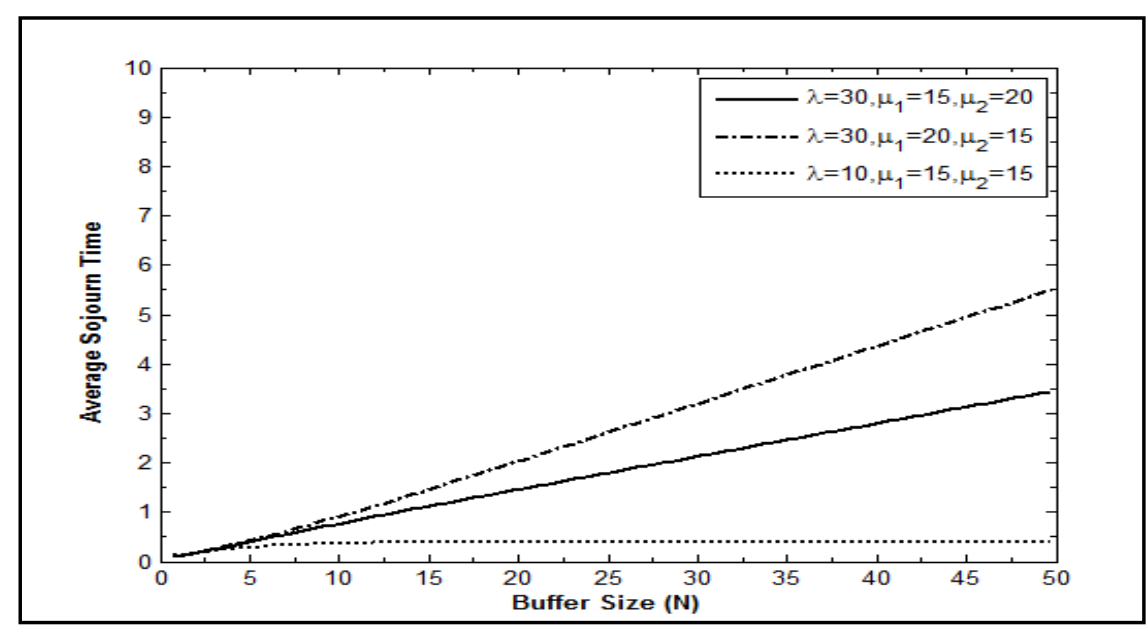

Figure 10. Average Sojourn Time against Buffer's size (N)

These findings mean that increasing the size of the buffer (allocated space for memory) will insure the success of transmitting all data even with latency, however this is not always feasible especially in wireless sensor networks due to energy and hardware limitations.

5.2.2. The effect of arrival rate $\lambda$ : the effect of arrival rate of on blocking probability is represented in figure 11 in which, the blocking probability increases in line with the increase observed in $\lambda$. For small values of $\lambda$, the blocking probability of the system is considered zero as the packets are served as soon as they arrive. However, as $\lambda$ gets higher than the service rate, the blocking and packets will in turn get dropped as soon as the buffers are full.

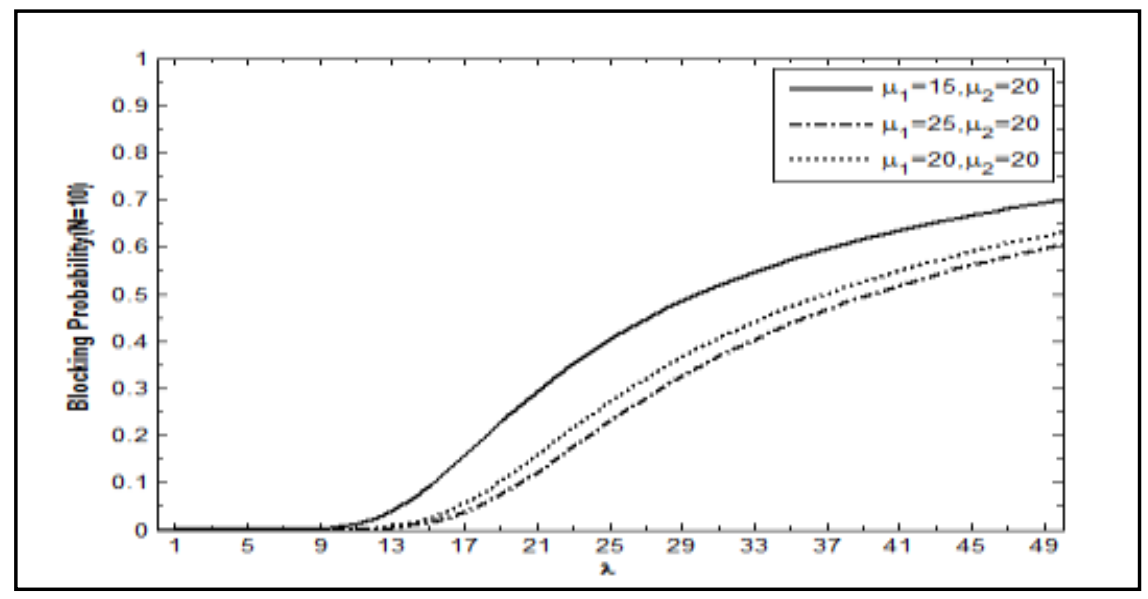

Figure 11. Blocking Probability as a Function of Arrival Rate $(\lambda)$

The highest blocking probability will be for the lowest service rate at server 1 as considering that $\lambda$ will be higher than $\mu$ most of the time. Increasing the value of $\mu_{1}$ will achieve better performance of the system.

The effect of arrival rate on the average number of packets in the system is shown in figure 12. Any increase observed in the arrival rate may equally increase the average number of packets for the same buffer's size. 


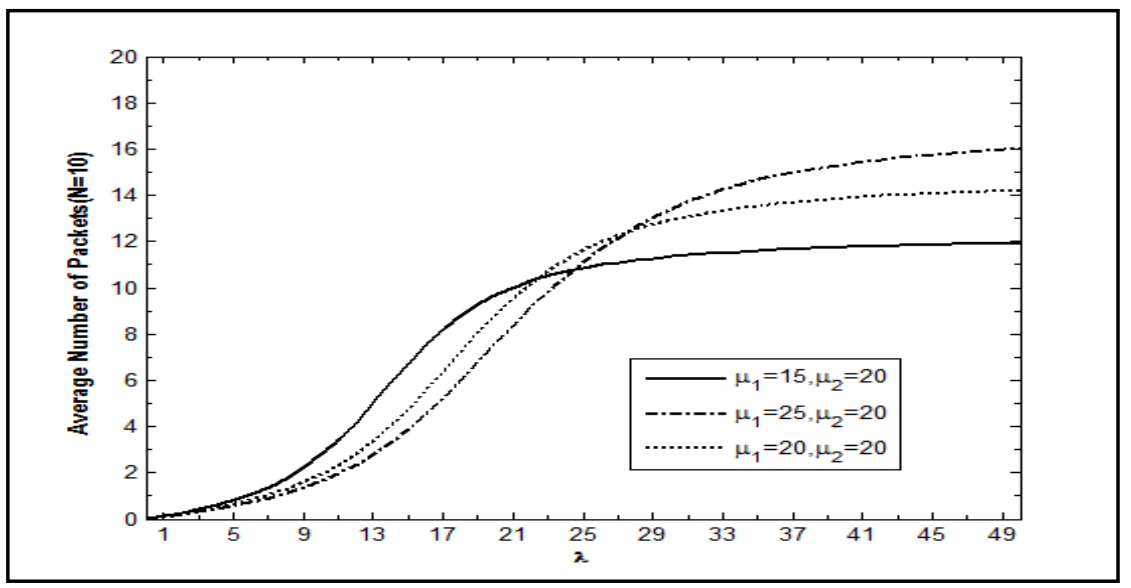

Figure 12. Average Number of Packets in the System vs. Arrival Rate $(\lambda)$

On the other hand, for lower values of arrival rates, in which $\lambda<\mu$, the highest number of packets can be achieved for the lowest value of $\mu$.As the packets are served at relatively a higher rate than the arrival rate, therefore, it is more likely that a lower service rate may result in more packets being presented in the buffer. As $\lambda$ increases to be more than $\mu$, the buffers may also be full and the arrival rate at server 2 can be equally high too. Therefore, for values of which $\mu_{1}>\mu_{2}$, the highest average number of packets can be achieved.

The average total time spent by a packet in the system is shown in figure 13. In general, the average time may increase in line with the increase observed in $\lambda$. However, it also depends on the value of service rate especially at server1, therefore, the lower service rate is relatively the longer time is spent by each packet in the system.

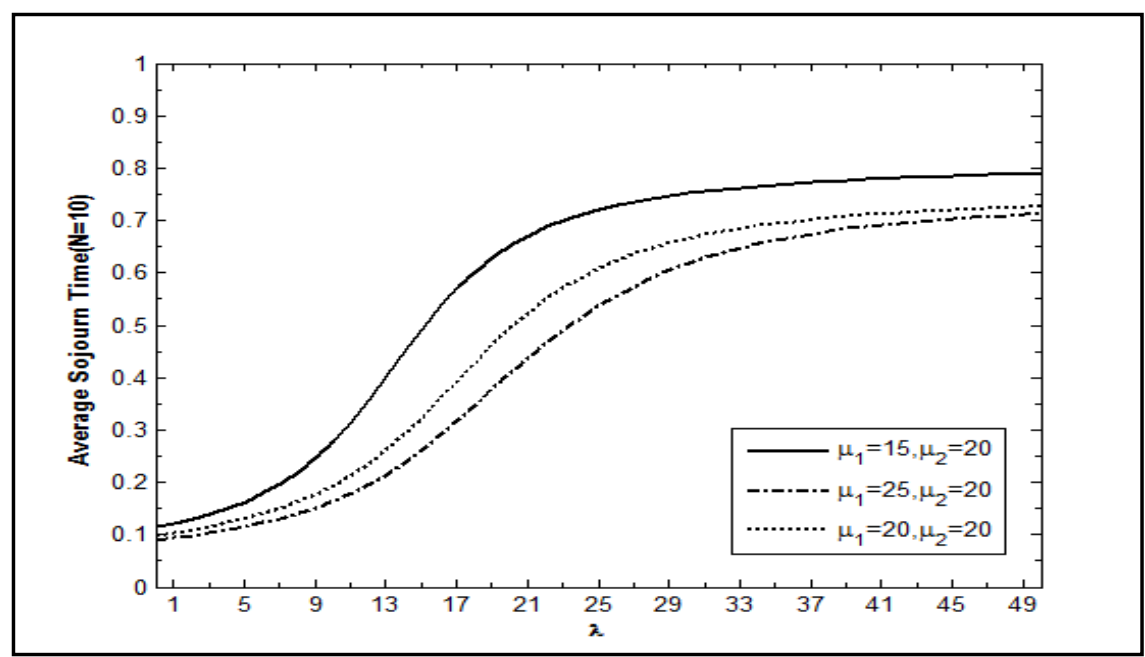

Figure 13. Average Sojourn Time in the System vs. Arrival Rate $(\lambda)$

The implication of the effect of arrival rate of packets on the system's performance can be summarized into two points:

The system will be never blocked will be never blocked when the arrival rate of packets into the system are less than the services rates of all servers in that system and the time spent by a packet in the system will be at the minimum.

Increasing the arrival rate of the system will increase its utilization and more packets can be found in the system. So by increasing the number of date collected by sensor there 
will be more data to be processed and transmitted. When the number of packets enter the system become high there will be data loss.

It is known that the time a unit spends in a server for processing rely upon the number of waiting units besides the speed of the processor. For instance, it is understood that the time needed to run a program on a pc is subject to the amount of programs running. At that point, in this section, we assume that the service times are still exponentially distributed, however the sojourn time spent in the system depends also on the number of packets in the queue.

5.2.3. The Effect of Service Rate at Server $1 \boldsymbol{\mu}_{1:}$ the effect of service rate at server 1 on the system's blocking probability is shown in figure 14. The trend of the graph can be divided into two sections. The first section in which $\mu_{1}<\mu_{2}$ and $\lambda>\mu_{1}$, the blocking probability will decrease as the packets served at a relatively higher rate than it originally arrived. However, for the case in which $\mu_{1}>\mu_{2}$, the blocking probability increases in line with the increase observed in $\mu_{1}$ because both the values of $\lambda$ and $\mu_{1}$ are considered greater than $\mu_{2}$.

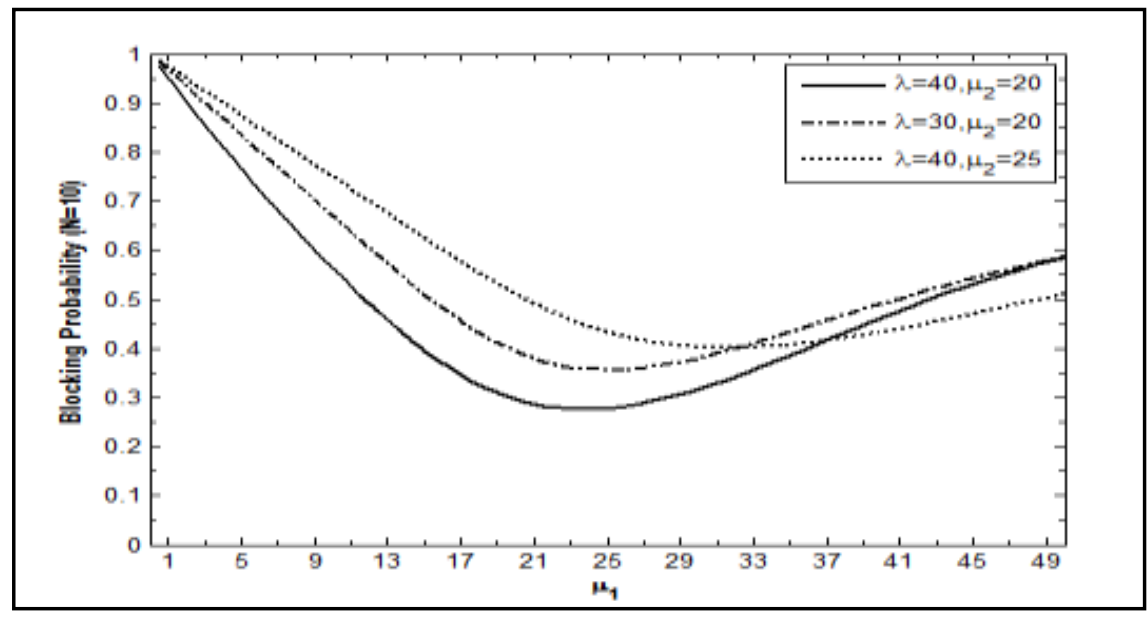

Figure 14. Blocking Probability vs. Processing Unit's Service Rate $\left(\mu_{1}\right)$

The effect of increasing $\mu 1$ on average number of packets is shown in figure 15 . The number of packets for $\mu_{1}$ being less than the buffer's size is limited to the first server's buffer's size, but it will slowly increase due to the increase of service rate at server 1 and accordingly the arrival rate at server 2 . When $\mu_{1}$ becomes higher than $\lambda$, the number of packets at server 1 will decrease. Although the number of packets at server 2 will increase but not with a similar rate.

The average sojourn time as a function of $\mu_{1}$ is illustrated in figure 16. An increase in the service rate may decrease the time spent by packets in the system till it reaches a constant and a very small value. 


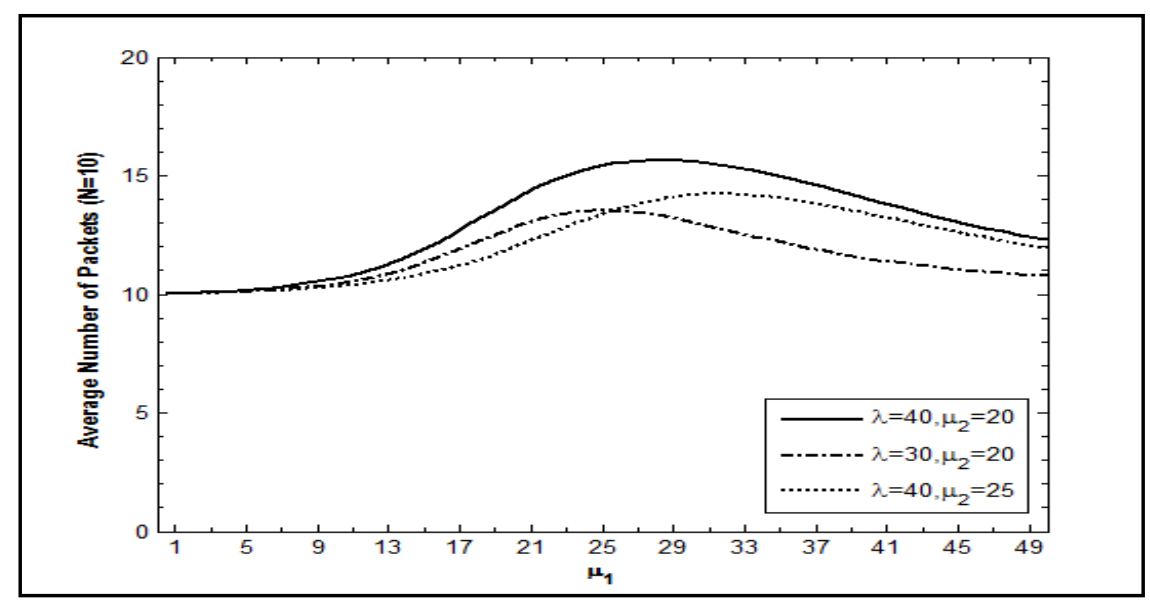

\section{Figure 15. Average Number of Packets as a Function of Processing Unit's Service Rate $\left(\mu_{1}\right)$}

As for sensing node in wireless sensor networks, increasing the processing unit's service rate will decrease the loss of data while the service rate of transmitting unit is constant and the arrival rate of data is greater than the service rate of both the processing and transmitting units. As the serving rate at processing unit becomes greater than the rate of data arriving, there will be data loss at the transmitting unit, as the processing's service rate is higher than the transmitter's service rate.

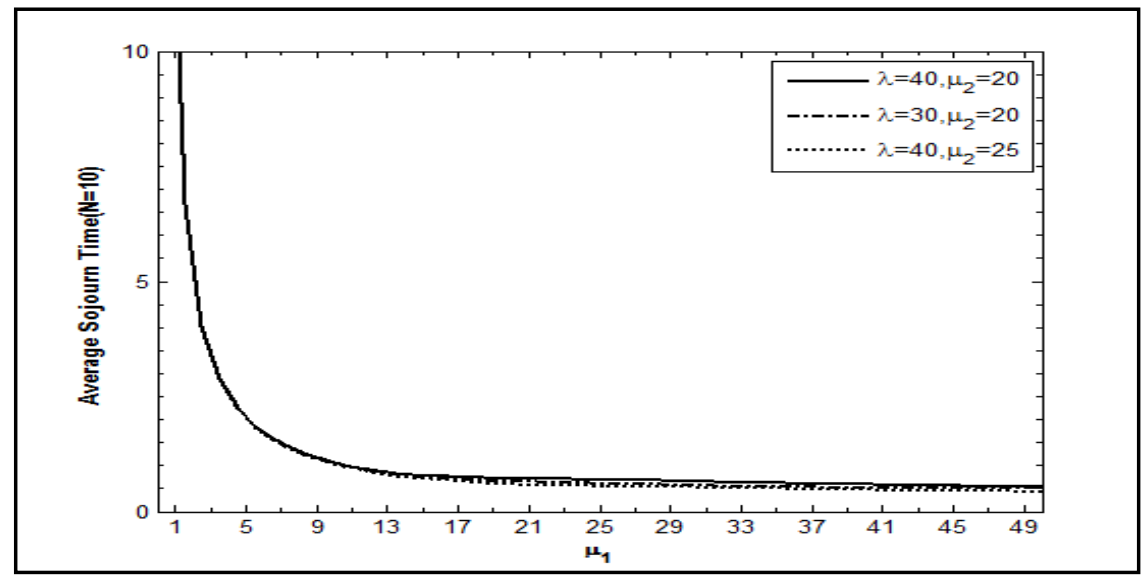

Figure 16. Average Sojourn Time in the System against Processing Unit's Service Rate $\left(\mu_{1}\right)$

5.2.4. The effect of service rate at server $2 \boldsymbol{\mu}_{2:}$ the effect of service rate at server $2\left(\mu_{2}\right)$ on blocking probability is shown in figure 17. It can be realized that the blocking probability decreases in accordance with the increase observed in $\mu_{2}$ till it reaches a constant value of around $\mu_{2}=\mu_{1}$. In other words, the higher the transmitter's service rate is, the less data loss occurred in system. When the service rate of both processing and transmitting unit is higher than the arrival rate, data loss will be at minimum. 


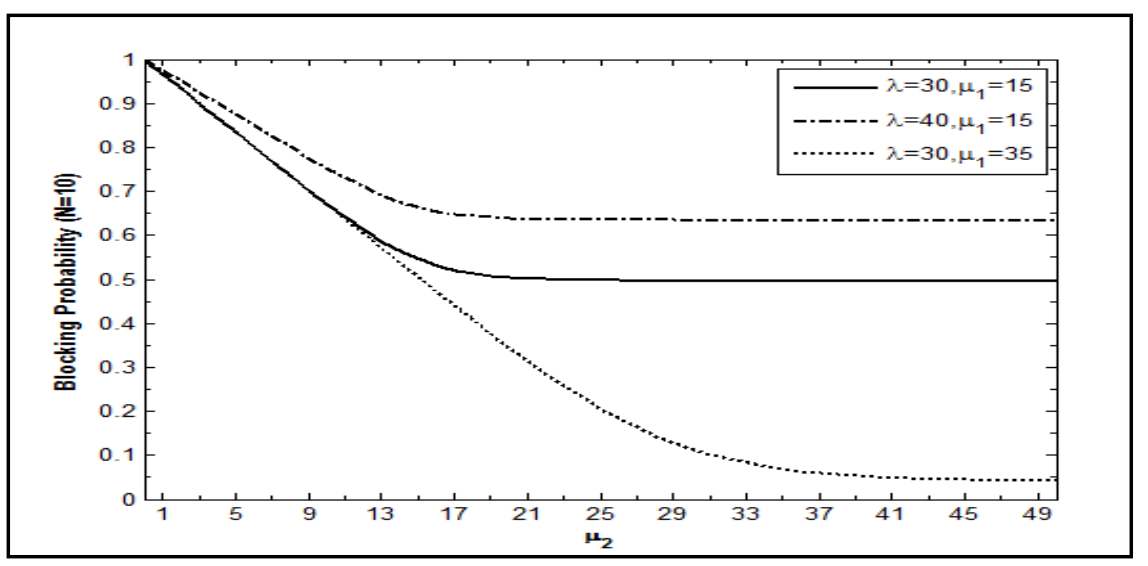

Figure 17. Blocking Probability against Transmitting Unit's Service Rate $\left(\mu_{2}\right)$

For small values of $\mu_{2}$, the average number of packets for $\mu_{1}<\lambda$ is almost the sum of buffer's size at each server as shown in figure (18).

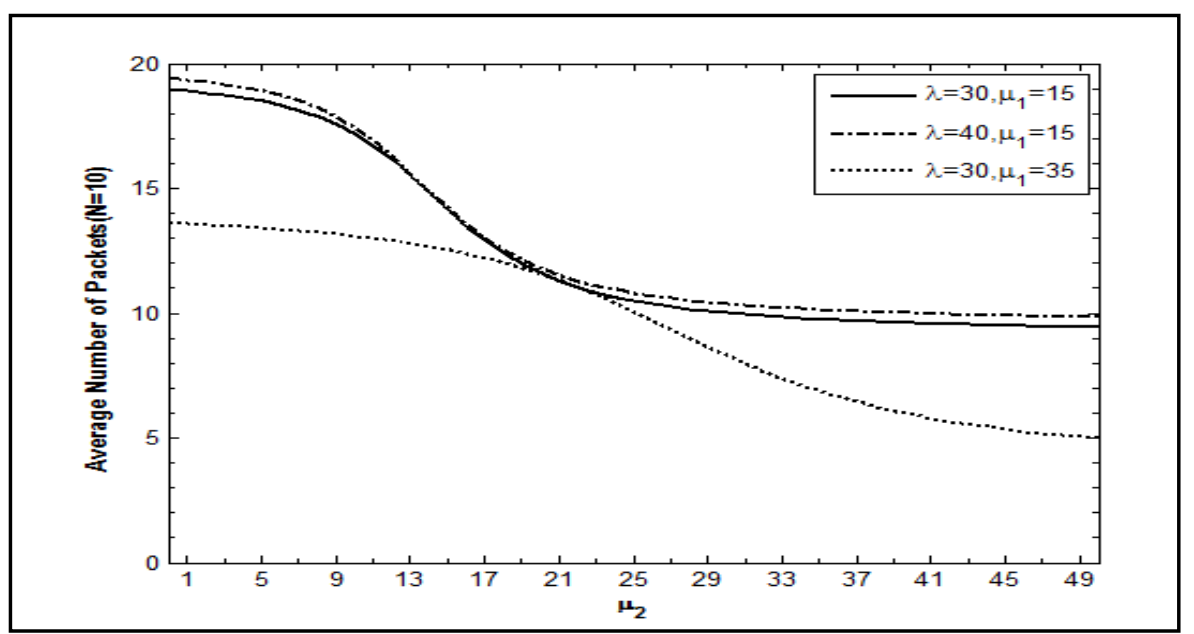

\section{Figure 18. Average Number of Packets in the System against Transmitting Unit's Service Rate $\left(\mu_{2}\right)$}

It has to be noted this is not the condition in which $\mu_{1}>\lambda$ as most packets are found at server 2 which just has a low server rate. As $\mu_{2}$ gets higher, the service rate at server 2 will equally increase which in turn will result in lesser packets being available at server 2 . Such a case will reduce the effect of server 1 has a low service rate and more packets. For values of $\mu_{1}>\lambda$, any increase observed in $\mu_{2}$ will decrease the average number of packets even further.

Increasing the value of $\mu_{2}$ may accordingly decrease the average time of a packet in the system as shown in figure 19. A relatively higher service rate at server 2 will in turn makes a packet spend lesser time in the system than that of the lower service rates. 


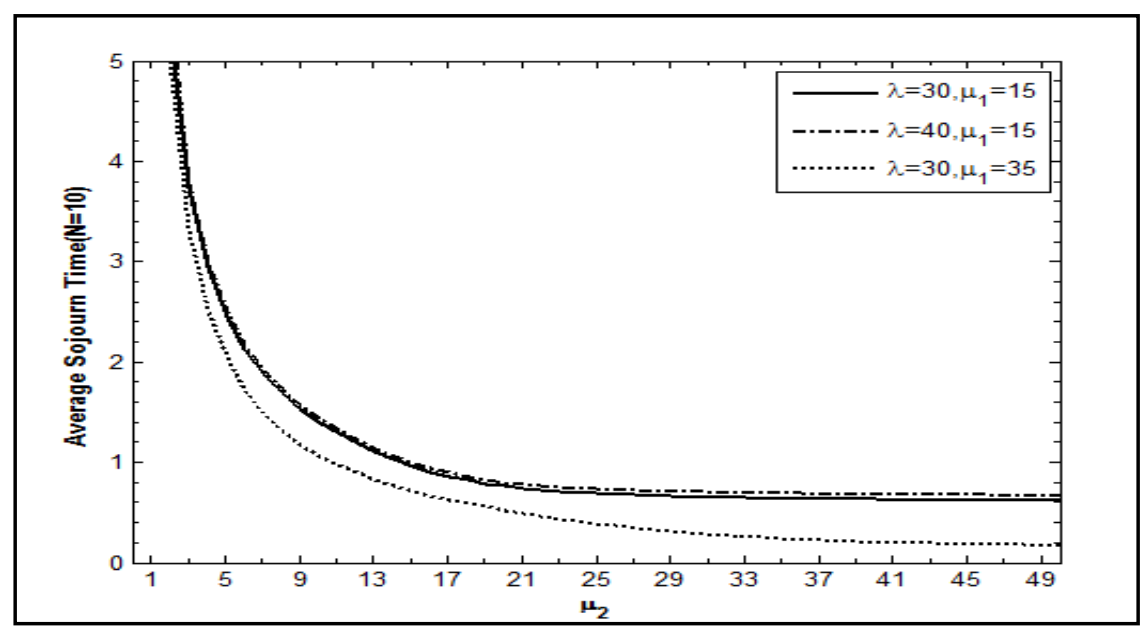

\section{Figure 19. Average Sojourn Time as a Function of Transmitting Unit's Service Rate $\left(\mu_{2}\right)$}

5.2.5. The effect of service rate $\left(\mu_{1}=\mu_{2}=\mu\right)$ : when the service rate at the first server equals the service rate at the second server as can be seen in figure 20, the blocking probability decreases as the $\mu$ increases. For values of which $\mu$ is higher than the $\lambda$, the blocking probability will therefore become a constant value. This case is the optimum case where data is processed at equal rate in both units. thus at high service rate all packets entering the system will be processed as soon as it arrives and then it will be capsulated and prepared for transmitting with minimal delay and data loss.

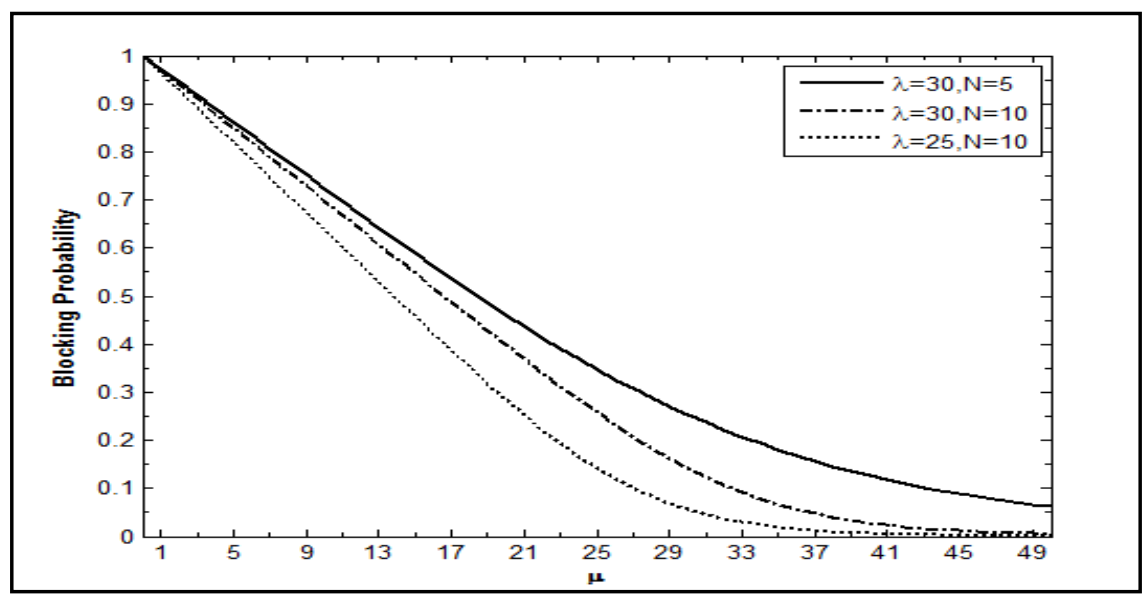

Figure 20. Blocking Probability against Service Rate $(\mu)$

The average total time of a packet in the system decreases as the value of $\mu$ is observed to increase. The graph in figure 21 indicates an interesting trend of the total time utilized in the system. As there is a small leap corresponding to the fact that in the event of $\mu$ equals zero, there will not be any arrival at server two thus making the total time equivalent to zero. 


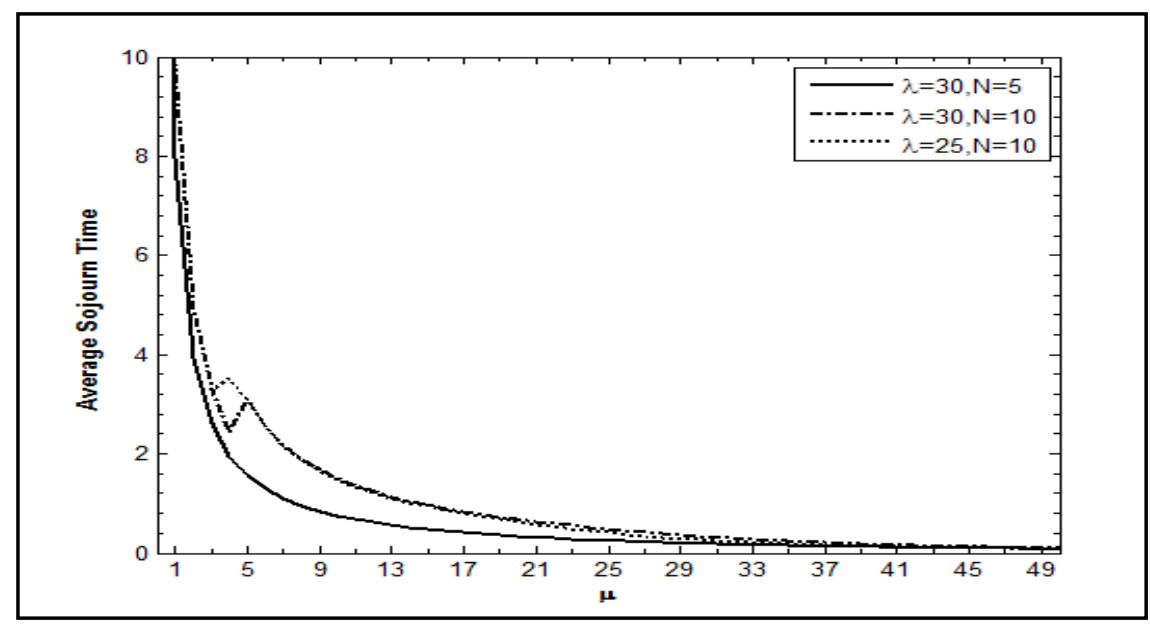

\section{Figure 21. Average Sojourn Time in the System against Service Rate $(\mu)$}

At the same time, the service time at server 1 which equals $1 / \mu$ will be equivalent to infinity, as $\mu$ is observed to increase at server 1 but still there are no packets at server 2 till packets served at server 1 arrives at server 2 and in such a case, the sojourn time will be its service time along with the queuing time.

Figure 22 describes the effect of increasing the $\mu$ on the average number of packets in the system. When $\mu$ equals zero or slightly greater than that, the number of packets in system will be equivalent to the buffer's size at server 1 due to the fact that there is no processing service at server 1 . Therefore, no packets may leave that server and enter server 2 either. Any increase observed in $\mu$ will in turn result in the number increasing to values of (the size of buffer $1+1 / 2$ the size of buffer 2 ). It is only after that it may start decreasing again due to the increase of service rate of both servers.

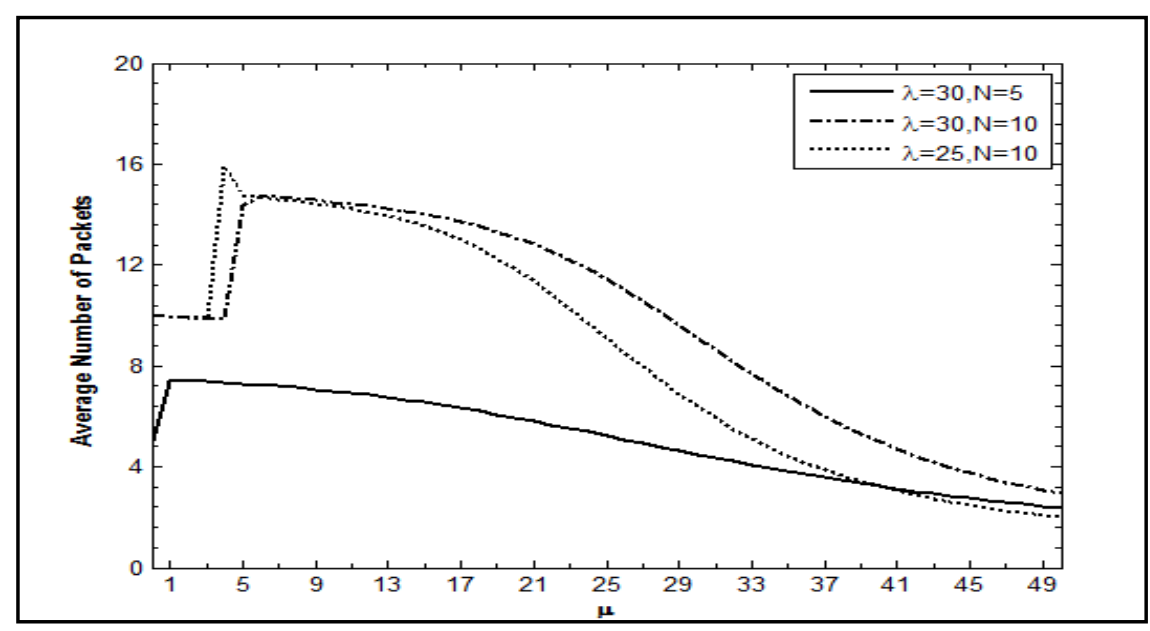

Figure 22. Average Number of Packets in the System vs. Service Rate $(\mu)$

5.2.6. The effect of service rate1/ service $\operatorname{rate} 2\left(\mu_{1} / \mu_{2}\right)$ : this scenario is designed to study the case where the service rate of one of the servers' service rate is kept fixed while varying the other server's service rate. Increasing the service rate of one of the servers always results in better performance. However, in wireless sensor networks there are 
constraints of cost, size and energy and increasing one server's serving capabilities limitlessly is unfeasible. The model displays beyond which point increasing the service rate becomes a waste of money and energy as the blocking probability becomes constant no matter how much the service rate of processing or transmitting unit is incremented. Figure 23 illustrates how any increase observed in $\mu_{1} / \mu_{2}$ will affect the blocking probability of the system. As an increase in $\mu_{1} / \mu_{2}$, the blocking probability will in turn decrease.

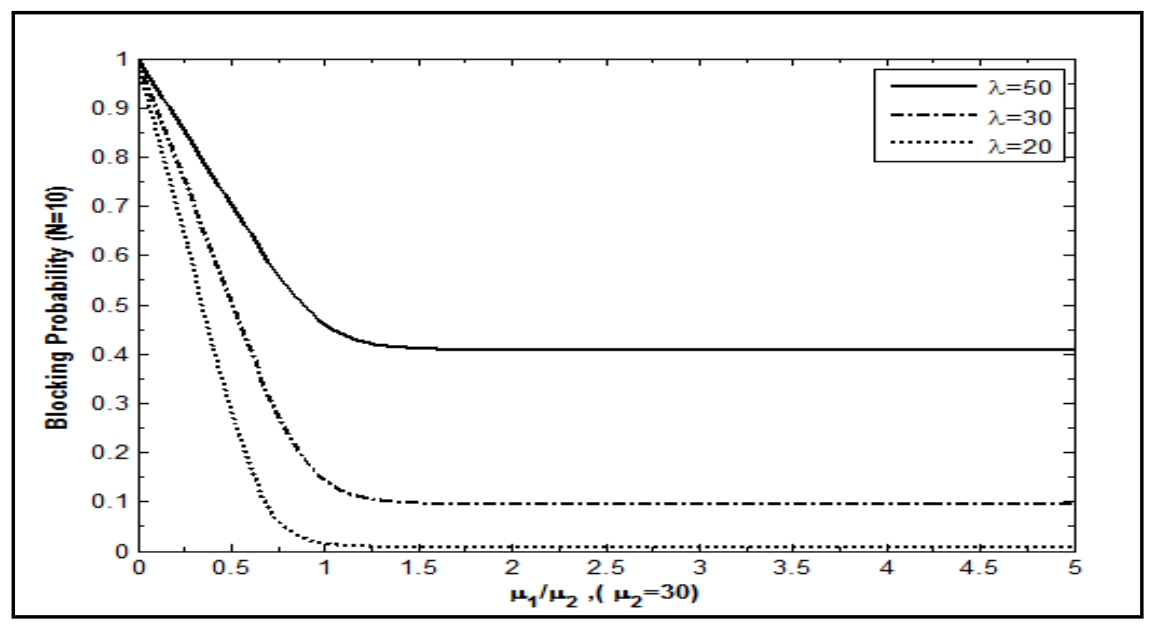

Figure 23. Blocking Probability against $\mu_{1} / \mu_{2}\left(\mu_{2}=30\right)$

This is due to the increase in service rate ratio will imply that the increase observed in the value of $\mu_{1}$. In this regard, when $\mu 1$ increases, the blocking probability will notably decrease significantly for values of $\mu_{1}<\mu_{2}$ owing to the fact that although there is blocking in server1, there will not be any blocking found in server 2 but when $\mu_{1} / \mu_{2}$ increases beyond 1 , the blocking probability will become a constant value as the decrease in blocking probability of server 1 faces an increase in blocking probability in server 2 .

The effect of $\mu_{1} / \mu_{2}$ on the average sojourn time is illustrated in figure 24 . As $\mu_{1} / \mu_{2}$ increases, the average time of a packet in the system will decrease. The highest value for average sojourn time is when the arrival rate at the highest value. Because when more packets start to arrive, will result in more time the packets spend waiting for their services.

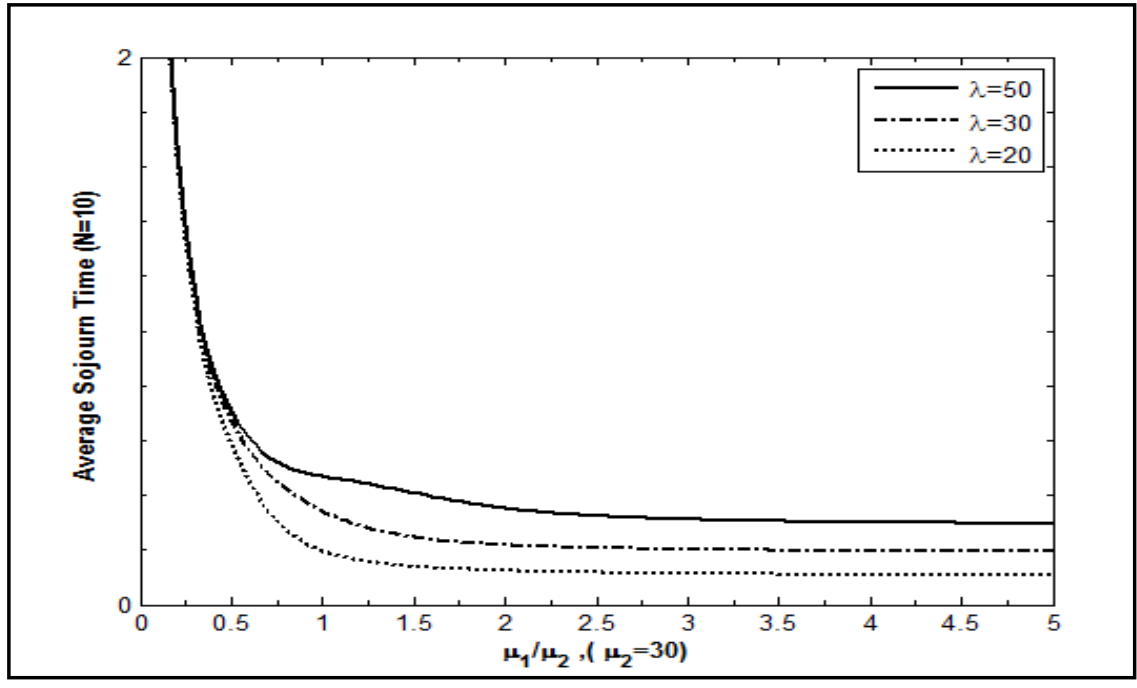

Figure 24. Average Sojourn Time in the System Vs. $\mu_{1} / \mu_{2}\left(\mu_{2}=30\right)$ 
For values of $\mu_{1}>\lambda$, the arrival rate of server 2 can be equivalent to $\lambda_{1}$. Therefore, any increase observed in $\mu_{1}$ for constant $\mu_{2}$ will affect server1's total time only and this the reason for why the value of sojourn time becomes constant.

In order to study the effect of $\mu_{1} / \mu_{2}$ on the average number of packets in the system, three values of $\lambda$ where selected; one being larger than $\mu_{2}$ while the other equals $\mu_{2}$ and the third when $\lambda<\mu_{2}$ for the same buffer's size of 10 as shown in figure 25 . When $\lambda>\mu_{2}$, the average number of packets will increase up to the value in which $\mu_{1}=\lambda$, accordingly the service rate at server1 gets higher and the buffer will not be packed so the number of packets in the system will decrease instead.

For $\lambda=\mu_{2}$, the average number is constant for small values of $\mu_{1}$, however as $\mu_{1}$ increases, the average number of packets will significantly decrease. The most noticeable decrease observed in the average number of packets is when $\mu_{1}$ increases for $\lambda=20$ in which, the average number of packets reaches smaller values as the system becomes ideal for a longer time.

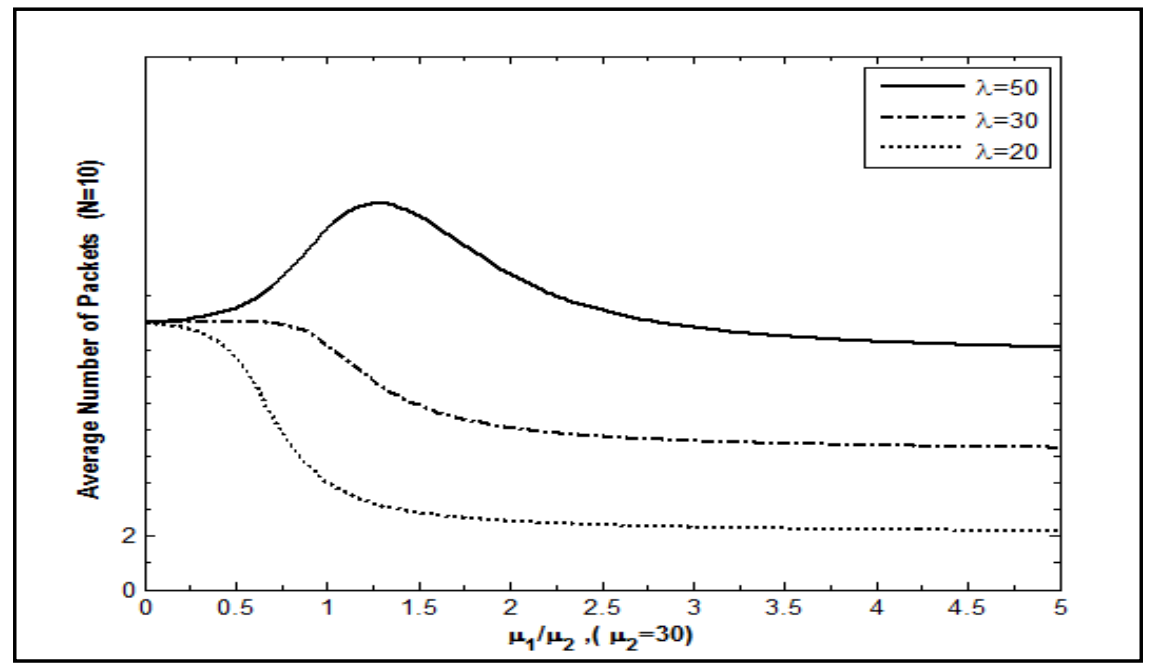

Figure 25. Average Number of Packets in the System Vs. $\mu_{1} / \mu_{2} \quad\left(\mu_{2}=30\right)$

\section{Conclusion}

In this paper, a Markovian chain queuing model for wireless sensor networks was introduced. Considering the discussion in the foregoing sections, the conclusions which can be drawn are:

[1] WSN designs which have limited resources to utilize, it is necessary for them to be supported with buffers which are capable of accommodating to the number of data units which enter the system;

[2] In the event of the average service rate of wireless transmission being equal to that of the data processing rate, and the data processing rate is 5 times greater than the sensor data arrival rate, the rate of data loss for the WSN node can therefore be almost zero (less than 0.0001);

[3] A reasonable allocation of model structure parameters and data processing rate will help improve the system's overall performance under the conditions of resources being rather limited;

[4] Although an increase in both the service rate of the data processing unit along with the constant data transmission unit's service rate which result in a decrease in the blocking probability, the blocking probability will remain constant upon reaching a specific point. Therefore, any increase in the service rate of one of the servers beyond that point is considered wasting the resources available (it is mainly when $\mu_{1}$ equals $\mu_{2}$, given 
that both of the services rates are at least 3 times higher than that of the sensor's data rate )

[5] The size of the buffer being greater will ensure that the lesser the blocking probability which the system suffers from. In this regard, with a relatively larger buffer capacity, the system approaches the performance of $\mathrm{M} / \mathrm{M} / 1$ queuing model (one server model with infinity waiting space) with almost a zero blocking probability. However, such a scenario can be considered too ideal for wireless sensor networks with limited amount of hardware and power supply.

\section{Acknowledgments}

This work is partially funded by International Islamic University Malaysia RMC grant No.: RIGS16-362-0526

\section{References}

[1] J. Yick, B. Mukherjee and D. Ghosal, "Wireless sensor network survey", Computer networks, vol. 52, no. 12, (2008), pp. 2292-2330.

[2] A. Rustamov, "Measurement of QoS in wireless sensor networks with single multimedia traffic-class", in Problems of Cybernetics and Informatics (PCI), 2012 IV International Conference, (2012), pp. 1-4.

[3] M. Matin and M. Islam, "Overview of wireless sensor network: INTECH Open Access Publisher", (2012) DOI: 10.5772/49376 ISBN 978-953-51-0735-4, Published: September 6, 2012.

[4] A. Fallahi, E. Hossain and A. S. Alfa, "QoS and energy trade off in distributed energy-limited mesh/relay networks: A queuing analysis", IEEE Transactions on Parallel and Distributed Systems, vol. 6, no. 17, (2006), pp. 576-592.

[5] B. Tang, R. Bagai, F. Nilofar and M. B. Yildirim, "A Generalized Data Preservation Problem in Sensor Networks-A Network Flow Perspective", in the 8th International Workshop on Wireless Sensor, Actuator and Robot Networks (WiSARN 2014), Benidorm, Spain, (2014).

[6] J. Luo, L. G. Jiang and C. He, "Finite queuing model analysis for energy and QoS tradeoff in contention-based wireless sensor networks", in the International conference on communication (ICC), IEEE Communications Society, (2007), pp. 1-6.

[7] D. Chen and P. K. Varshney, "QoS Support in Wireless Sensor Networks: A Survey", in the International conference on wireless networks, (2004), pp. 1-7.

[8] M. Abo-Zahhad, O. Amin, M. Farrag and A. Ali, "A Survey on Protocols, Platforms and Simulation Tools for Wireless Sensor Networks", Int. Journal Energy, Inf. Commun, vol. 5, no. 6, (2014), pp. 1734.

[9] G. H. Li, C. M. Zhu and X. Li, "Application of chaos theory and wavelet to modeling the traffic of wireless sensor networks", in 2010 International Conference on Biomedical Engineering and Computer Science, (2010), pp. 1-4.

[10] M. Escheikh and K. Barkaoui, "Opportunistic MAC layer design with stochastic Petri Nets for multimedia ad hoc networks", Concurrency and Computation: Practice and Experience, vol. 22, no. 10, (2010), pp. 1308-1324.

[11] B. Lacerda and P. U. Lima, "Petri nets as an analysis tool for data flow in wireless sensor networks", in 1st Portuguese Conference on WSNs, Coimbra, Portugal, (2011), pp. 1-6.

[12] A. W. Liehr and K. J. Buchenrieder, "Simulating inter-process communication with extended queuing networks", Simulation Modelling Practice and Theory, vol. 18, no. 8, (2010), pp. 1162-1171.

[13] N. Bisnik and A. A. Abouzeid, "Queuing network models for delay analysis of multihop wireless ad hoc networks", Ad hoc Networks, vol. 7, no. 1, (2009), pp. 79-97.

[14] G. Degirmenci, J. P. Kharoufeh and O. A. Prokopyev, "Maximizing the lifetime of query-based wireless sensor networks", ACM Transactions on Sensor Networks (TOSN), vol. 10, no. 4, (2014), article no. 56.

[15] T. Qiu, L. Feng, F. Xia, G. Wu and Y. Zhou, "A packet buffer evaluation method exploiting queueing theory for wireless sensor networks", Comput. Sci. Inf. Syst., vol. 8, no. 4, (2011), pp. 1028-1049.

[16] W. S. Jang, D. E. Lee and J. H. Choi, "Ad-hoc performance of wireless sensor network for large scale civil and construction engineering applications", Automation in Construction, vol. 26, (2012), pp. 32-45.

[17] N. Kayastha, D. Niyato, E. Hossain and Z. Han, "Smart grid sensor data collection, communication, and networking: a tutorial", Wireless communications and mobile computing, vol. 14, no. 11, (2014), pp. 1055-1087.

[18] T. Qiu, F. Xia, L. Feng, G. Wu and B. Jin, "Queueing theory-based path delay analysis of wireless sensor networks", Advances in Electrical and Computer Engineering, vol. 11, no. 2, (2011), pp. 3-8.

[19] M. Usman, H. C. Yang and M. S. Alouini, "Extended delivery time analysis for cognitive packet transmission with application to secondary queuing analysis", IEEE Transactions on Wireless Communications, vol. 14, no. 10, (2015), pp. 5300-5312. 
[20] M. Kamali, L. Laibinis, L. Petre and K. Sere, "Formal development of wireless sensor-actor networks", Science of Computer Programming, vol. 80, (2014), pp. 25-49.

[21] L. Mokdad, J. Ben-Othman, B. Yahya and S. Niagne, "Performance evaluation tools for QoS MAC protocol for wireless sensor networks", Ad hoc Networks, vol. 12, (2014), pp. 86-99.

[22] A. Melikov and A. Rustamov, "Queuing management in wireless sensor networks for QoS measurement”, Wireless Sensor Network, vol. 4, no. 9, (2012), pp. 211-218.

[23] V. C. Gungor and G. P. Hancke, "Industrial wireless sensor networks: Challenges, design principles, and technical approaches", IEEE Transactions on Industrial Electronics, vol. 56, no. 10, (2009), pp. 42584265.

[24] S. Tang and W. Li, "QoS supporting and optimal energy allocation for a cluster based wireless sensor network", Computer Communications, vol. 29, no. 13, (2006), pp. 2569-2577.

[25] J. Liu and T. Lee, "A framework for performance modeling of wireless sensor networks", Paper presented at the IEEE International Conference on Communications, 2005, ICC, (2005).

[26] Q. Li, M. Yang, H. Wang, Y. Jiang and J. Zeng, "A finite queue model analysis of PMRC-based wireless sensor networks", in Proceeding ICWN, (2008).

[27] W. Guo-guang, L. Gui-xiong and Z. Song-bin, "Modeling and Dynamic Performance Evaluation of WTIM Based on Petri Nets", Journal of South China University of Technology (Natural Science Edition), vol. 41, no. 9, (2013), pp. 108-112.

[28] S. M. Ross, Introduction to probability models: Academic press, (2014). 